\title{
L'INFERNO SULLA SCENA MEDIOEVALE: I CASI ITALIANO E FRANCESE
}

\author{
Federica Natta
}

\begin{abstract}
The paper aims to focus on the evolution of the infernal theme in Italian and French theatrical contexts in the period between the $14^{\text {th }}$ and the $15^{\text {th }}$ centuries. Specifically, we have examined three types of sources: drama, hymns and sacred late medieval iconography of hell.

Keywords: hell; scene; lauda; mysteries; sacred representations.

Abstract: Il saggio si propone di mettere a fuoco lo sviluppo del tema infernale nei contesti teatrali italiano e francese in un periodo compreso tra il XIV e il XV secolo. Nella fattispecie, si sono prese in considerazione tre tipi di fonti documentarie: testi drammatici, laudi sacre e iconografie infernali tardo medievali.

Parole chiave: inferno; scena; lauda; misteri; sacre rappresentazioni.
\end{abstract}

Nel teatro medioevale la rappresentazione dell'Inferno come luogo di punizione dei peccatori ha un ruolo limitato. Spesso, infatti, esso è solo un elemento obbligato del dispositivo drammatico ma non è lo scenario di vere e proprie rappresentazioni.

In effetti, i temi privilegiati dell'ambito teatrale, la Passione o le Vite dei Santi, non si prestano allo sviluppo di questo aspetto e anche l'episodio della Discesa al Limbo, non comporta che tardivamente un'evocazione della sorte dei dannati. Infine il Giudizio Universale, nel quale l'Inferno è suscettibile di essere trattato come tema a sé stante, è messo in scena molto raramente (Sheingorn 1985).

\section{Le laudi drammatiche italiane}

Nate nel contesto delle confraternite umbre del XIII secolo, in particolare in rapporto al movimento dei Disciplinati del 1261, le laudi sono in origine solamente narrative; solo successivamente danno luogo a delle vere e proprie rappresentazioni realizzate dalle stesse confraternite.

In questa tradizione italiana, il soggetto infernale è in stretta relazione al tema del Giudizio, la cui comparsa è repentina: lo si incontra già nelle prime raccolte, in particolare nel Laudario di Cortona, datato alla fine del XIII secolo. ${ }^{1}$ Il racconto, però, non si allontana

\footnotetext{
1 Lauda n. 34 (ed. Varanini-Banfi-Ceruti Burgio 1981). A uso della confraternita di Santa Maria delle Laude, e non dei Disciplinati, si tratta della più antica raccolta conservata. Di carattere puramente narrativo, la lauda comprende 60 versi.
} 
molto dal testo evangelico di Matteo; non comporta che un'allusione discreta al supplizio e termina con un appello rivolto agli eletti («Venite a regno delitioso»).

Nel Laudario di Pisa invece, composto tra il 1305 e il 1315, una lauda del giudicio riserva maggior importanza alla sorte dei reprobi. ${ }^{2}$ Lo sviluppo della forma dialogica, in particolare, permette di mostrare i dannati nell'atto di implorare il perdono divino mentre il Cristo Giudice fa menzione, nella sentenza di condanna, di numerose categorie di peccatori (sodomiti, usurai, ladri, meretrici e traditori).

E' solo però con il Laudario di Perugia che si ha una rappresentazione dettagliata del Giudizio.

È difficile datare con precisione la lauda intitolata L'Anticristo e il Giudizio finale ma il suo carattere nettamente drammatico suggerisce di situarne la composizione nel secondo quarto del XIV secolo. ${ }^{3}$ La sua rappresentazione era prevista per una delle domeniche dell' Avvento. ${ }^{4}$ Il debutto avviene con sei strofe consacrate all'Anticristo ma l'essenziale della lauda concerne soprattutto il tema del Sentenza Finale. ${ }^{5}$ Diciannove strofe ne sviluppano gli episodi: la Risurrezione, l'Esibizione delle Piaghe e dei simboli della Passione, la Divisione delle anime (Partage), i Verdetti di Condanna e di Elezione. Già in questa fase appare un disequilibrio tra i due gruppi: se tre strofe sono sufficienti per l'annuncio della sorte degli eletti e la loro risposta, lo scambio verbale con i dannati ne richiede sette. ${ }^{6}$

Seguono poi trenta strofe interamente consacrate ai peccatori: c'è l'appello alla Vergine affinchè interceda per loro presso il Figlio diletto, ${ }^{7}$ poi, attraverso le richieste di clemenza dei dannati, sono evocati gli aspetti essenziali della loro condizione futura.

Infine, questa parte si conclude con una lunga tirata, nella quale il Cristo reitera la sentenza di condanna, rimarcando l'inutilità del pentimento tardivo (perché post mortem). Ma in luogo di rivolgersi all'insieme dei reprobi, come Matteo, pronuncia delle condanne specifiche per sei dei sette vizi capitali (vv. 283-318). Si menzionano i golosi, gli avari, i lussuriosi, gli accidiosi, i superbi e gli iracondi: il Cristo precisa il supplizio al quale sono destinati e indica il demone deputato a eseguire la punizione. ${ }^{8}$ Anche se

2 Lauda n. 64 (ed. Staaff 1931). Si tratta della prima raccolta conservata ad uso dei Disciplinati. Contiene delle laudi narrative nelle quali lo sviluppo dei passaggi dialogici è oltremodo sensibile. La Lauda 65 (Dell'anima dannata) mostra una monaca, ritenuta da tutti virtuosa, in mezzo alle pene infernali. Si illustra qui il tema della penitenza e il peccato di superbia ma non si ha alcuna descrizione infernale.

3 Questa lauda appare in due raccolte di Perugia (P: Perugia 955 e V: Valliceliana A26). L'analisi dei palinsesti ha premesso di stabilire che queste raccolte sono state entrambe costituite dopo il 1350 (Vinti 1950). Derivano tutte e due da un manoscritto più antico, probabilmente dell'inizio del XIV secolo. Tuttavia contengono anche delle laudi comuni più tarde come quella per la canonizzazione di S. Tommaso (posteriore al 1323). Infine Terrugia (1980: 459) constata che, se le prime utilizzazioni drammatiche di laudi risale all'inizio del XIV secolo, la creazione invece di testi propriamente teatrali (ed è il caso di cui ci si occupa) non interviene prima del quarto decennio di quel secolo. Tenuto conto della sua ampiezza, quest'opera deve essere considerata tra le più moderne e probabilmente è stata aggiunta a pièces più tradizionali di raccolte anteriori.

4 La versione del manoscritto P è edita da De Bartholomaies 1943. Quella del manoscritto V invece è edita da Galli 1910: 107 e ripresa da Bonfantini 1942. Le due raccolte sono organizzate secondo il calendario liturgico. La nostra lauda è introdotta dalla menzione «In Domenica de adventu» (senza dubbio la seconda che generalmente è associata alla Seconda Venuta di Cristo).

5 Nella versione P essa è costituita da 65 sestine (390 versi), in V, da 72 (432v).

6 Vv. 150-210. Si tratta di uno sviluppo del tema di Matteo, in particolare sull'attitudine nei confronti dei poveri.

7 La Vergine utilizza l'argomento materno («Per quel lacte ch'i te diei..») al fine di far revocare le sentenze già pronunciate («Io te prego, si esser puote/ Che la sententia tu revoche», vv. 233-234). Ma è troppo tardi: «Non è tempo d'appellare/la misericordia è mo' sospesa» (vv. 241-242).

8 Per esempio Satanasso per gli accidiosi, Belzebuthe per gli orgogliosi e Macomecto per gli iracondi. 
il settenario resta incompleto e le pene sono poco dettagliate, lo schema dei vizi capitali riceve, nel verdetto pronunciato dal Giudice, una legittimazione considerevole perché presentato come il fondamento di una differenziazione delle pene infernali.

I dannati sono successivamente chiusi nell'Inferno e la lauda termina con una scena nel corso della quale Lucifero e i suoi demoniaci sottoposti sono rappresentati nell'atto di disporre i supplizi per i diversi peccatori (vv. 337-390). I loro propositi sono meno strutturati rispetto a quelli del Cristo e non riprendono lo schema del settenario. Anche se si trova menzione dei golosi, degli avari, dei superbi (appesi per i capelli), sono usate qui soprattutto delle categorie più concrete. D'altra parte i tormenti sono meno precisi. ${ }^{9}$

Tuttavia, paragonata a quella di Cortona e di Pisa, questa lauda introduce una trasformazione sensibile grazie soprattutto agli elementi aggiunti al racconto evangelico. ${ }^{10} \mathrm{Cer}-$ te pene, inoltre, sono evocate con precisione, contemporaneamente la differenziazione delle categorie dei peccatori è molto sviluppata.

L'Anticristo e il Giudizio Finale integra, dunque, nella rappresentazione del Giorno Ultimo, una descrizione dettagliata e strutturata dell'Inferno che fa così, nel secondo quarto del 1300, il suo ingresso sulla scena dei drammi religiosi dell'Umbria. ${ }^{11}$

Comunque c'è da rilevare il fatto che una rappresentazione così precisa resta eccezionale, data l'epoca. La Raccolta di Assisi contiene una lauda iudicii, ma questa non riprende né la sentenza dettagliata del Cristo, né l'evocazione delle pene infernali. ${ }^{12}$ Se si ritrova il motivo delle suppliche dei dannati, tuttavia la pièce termina con l'appello agli eletti, invitati a prendere possesso del Regno Celeste, e con un'esortazione alla penitenza. Probabilmente occorre qui considerare una differenza sovente menzionata tra le laudi di Assisi e di Perugia: mentre le prime traggono dal loro legame con i Francescani un orientamento fortemente lirico, le confraternite di Perugia, soprattutto legate ai Domenicani, danno alle loro laudi una forma più drammatica e un contenuto più didattico (Baldelli 1962: 340).

Il Dicto dell'inferno, contenuto nella raccolta di una confraternita dell'Aquila, indica anch'esso i limiti della progressione del settenario. ${ }^{13}$

9 Gli omicidi e i traditori sono gettati in un pozzo; i superbi e i tiranni sono messi in una prigione piena di animali ripugnanti; i blasfemi hanno la lingua tagliata con una lama; i sodomiti sono «rostite a giusa de porchete». La categoria più sviluppata è quella dei falsi devoti e, in particolare, dei "falsi disciplinati" che fanno mostra di un'ipocrita penitenza. Essi sono rivestiti «d'una veste d'aspra penetentia» interamente infiammata. Questo indica chiaramente che la realtà della dannazione è possibile anche per i membri delle confraternite. Il tema infernale conosce dunque un doppio uso, interno ed esterno.

${ }_{10} \grave{E}$, tra l'altro, estremamente rilevante il fatto che le modifiche riguardino soprattutto i dannati. Il disequilibrio tra l'attenzione prestata agli eletti da una parte e ai peccatori dall'altra ha, in effetti, un qualcosa di stupefacente (tre strofe contro trentasette).

11 Le due raccolte di Perugia contengono anche un Contrasto del povero e del ricco, che segue lo schema della Parabola di Lazzaro (ed. Bonfantini 1942: 31-42). In realtà non si ritrova una vera e propria messa in scena dell'Inferno, ma i supplizi del ricco sono ben descritti («si faccia el macellare», «stemperate oro et argento, dateglielo per beveraggio», su questo testo vedere Lazar 1971a).

12 La lauda è composta di 198 versi; (vedere ed. Galli 1910: 199-204). Si tratta di una raccolta destinata alla confraternita di S. Pietro ad Assisi, senza dubbio della seconda metà del XIV secolo. In un'altra raccolta di Assisi, la celebre Illuminati (per la confraternita di S. Stefano, fondata nel 1324), nessuna lauda concerne il tema del Giudizio Finale.

${ }^{13}$ La pièce, ugualmente conosciuta sotto il nome di Contrasto del vivo e del morto (si tratta di un dialogo tra un defunto e un vivente) è edita da Percopo 1886. Essa figura in un manoscritto della fine del XV secolo ma data probabilmente al XIV. Il fatto di sapere se il morto subisce le pene dell'Inferno o del Purgatorio resta ambiguo. 
Il modello delle pene è quello di Onorio Augustodunense ma il principio del contrappasso è meno rigoroso. ${ }^{14}$

È il segno di uno sviluppo sensibile del principio di strutturazione ed è emblematico che il fuoco sia qui riservato ai lussuriosi. Infine, si nota che si tratta di una lauda interamente consacrata alle pene dell'aldilà.

Il tema del Giudizio Universale appare poi in sacre rappresentazioni del XV secolo che confermano la tendenza reperita nell' Anticristo e il Giudizio finale. Nella Rappresentazione del Di del Giudizio, composta nel XV secolo da Feo Belcari ${ }^{15}$ (D'Ancona 1872: III, 499$523)$, il settenario è utilizzato a due riprese: sulla scena del partage, un peccatore di ciascun gruppo dialoga con una rappresentazione della virtù opposta; poi, una volta nell'Inferno, un demone si rivolge, seguendo lo stesso ordine, a ciascuno dei dannati per annunciare la pena corrispondente. ${ }^{16}$ Ma se il settenario conosce qui un uso rigoroso, non si trova alcuna differenziazione delle pene: tutti i dannati indistintamente sono votati alle fiamme. Inoltre, il ruolo del sistema dei sette peccati capitali, si riduce: non solo non è più impiegato da Cristo ma la rappresentazione mette in scena anche dei gruppi definiti dal loro statuto sociale. ${ }^{17}$ Soprattutto questa rappresentazione realizza un profondo riequilibrio perché la forma dialogica permette, per due volte, di opporre i santi o le virtù ai gruppi di peccatori. Allo stesso modo, il verdetto divino relativo ai dannati e la risposta di questi, occupa lo stesso numero di strofe che l'episodio simmetrico concernente gli eletti: il Giudice sembra ritrovare la sua imparzialità.

Tuttavia, nonostante il dramma di Belcari prevalga per maggior equilibrio e rigore sulla lauda del secolo precedente, perde di essa l'intensità suppliziante che rappresentava un elemento di forte suggestione emotiva.

\section{I Misteri francesi}

Per quanto riguarda l'ambito drammatico francese si prende in considerazione un solo testo, rispettivamente il Jutgamen general (dramma provenzale, posteriore al $1481^{18}$ ), per la sua diffusione nel contesto italiano e per il legame con le laudi. ${ }^{19}$

Tuttavia è importante rilevare che l'opera si costituisce anche come un'eccezione rispetto allo stesso panorama francese del secolo XV: infatti essa rappresenta la prima versione autonoma e sviluppata del soggetto del Giudizio sulla scena.

14 Versi 155-254. L'acqua fredda è riservata agli accidiosi (freddezza nei riguardi di Dio), mentre draghi e serpenti attaccano gli invidiosi (così come anche i seminatori di discordia).

15 È la ripresa, ampliata, di un dramma di Antonio Araldo (prima metà del secolo). Sarà riutilizzato e riadattato, per esempio in una raccolta di Bologna, nel 1482: la Festa del Giudizio (De Bartholomaies 1943: III, 278-291).

${ }_{16}$ Questo doppio uso del settenario figura già nella versione di Araldo (De Bartholomaies 1943: III, 502).

17 Belcari aggiunge, durante il partage, una serie di discussioni tra ciascun gruppo e il Santo che avrebbe potuto essere un efficace protettore. Ma Pietro non può nulla contro il clero corrotto, né Francesco contro i poveri che credono che la loro condizione sia sufficiente a garantire la salvezza, né Girolamo per i penitenti ipocriti. Le stesse considerazioni valgono per il dialogo tra i mercanti eS. Nicola da Bari o tra le prostitute eS. Maria Maddalena.

${ }_{18}$ Datazione stabilita da Roy (1904) e ripresa da Lazar (1971: 42). Il testo deriva per una parte importante dalla traduzione francese del Processus Belial di Jacopo da Teramo.

19 La pièce importa anche per alcune modalità supplizianti che conoscono una diretta ripresa in certi figurazioni della serie ligure e per le quali non si è ancora individuato un referente testuale preciso. L'ipotesi deve essere ulteriormente approfondita ma a titolo di osservazione è interessante constatare la corrispondenza per certi supplizi: così la gola è tormentata da serpenti come nell'affresco di San Giorgio Campochiesa di Albenga, la lussuria è gettata in un calderone (Santuario della Madonna Bambina, Rezzo) una ruota è preparata per l'invidia e l'ira (tuttavia, quest'ultimo elemento punitivo ricorre in molti testi teatrali e non: lo ritroviamo, in effetti, anche in molte visioni dei secoli XII-XIII). 
In effetti, a partire dal XIII secolo, è la Passione di Cristo il tema per eccellenza dei Misteri e, tra l'altro, il soggetto non si presta a un'evocazione dell'Inferno. Di fatto, le Passioni del XIV secolo, si limitano all'episodio di Cristo che libera i Giusti dal Limbo e non contengono alcuna descrizione dell'aldilà dei dannati. Non è dunque nei Misteri che si può rinvenire una messa in scena del Luogo di Punizione. Il suo uso come strumento della giustizia divina trova più logicamente rapporto con il tema del Giudizio Universale ma questa tematica non conosce praticamente alcun sviluppo scenico se non che alla fine del XV secolo (Lazar 1971b: 11-13 e 36-45).

Esiste un testo intitolato Le Jour du Jugement, rappresentato nel 1397, ma esso è essenzialmente consacrato all' Anticristo. ${ }^{20}$ Non comprende alcuna descrizione dettagliata dell'Inferno e non aggiunge nulla alla struttura del testo di Matteo se non qualche episodio di dibattito davanti al Tribunale Celeste.

Eccezione a questa situazione è il dramma provenzale del Jutgamen.

Una prima distinzione dei peccatori si opera durante il partage: il Giudice pone rispettivamente alla sua sinistra i giudei, i pagani, il clero secolare, il clero regolare, i laici e gli uomini di legge. ${ }^{21}$ La seconda parte del dramma è un dibattito tra Misericordia e Giustizia, poi tra la Vergine e il Cristo; essa termina con il verdetto definitivo del Giudice che indica per tutti i peccatori la stessa condanna (vv. 976-1824).

Infine la terza parte è interamente dedicata all'Inferno. ${ }^{22}$ Essa è consacrata, in specifico, al castigo dei sette vizi capitali che, in forma di personaggi, sono rappresentati nell'atto di subire la loro punizione. ${ }^{23}$

Si tratta di supplizi la cui natura non è precisata ma, probabilmente, essa doveva essere particolarmente violenta, dato l'uso in scena di manichini (su questo punto Konigson 1975: 148-150).

Ciascun vizio riceve un tormento specifico: la Superbia è posta su un trono (la sedia, simbolo delle sue pretese e presunzioni terrene) e ingozzata con piombo fuso; l' Avarizia, a cui si rimprovera soprattutto la rovina di povera gente per la pratica dell'usura, è costretta a ingerire monete roventi; la Gola è tormentata da serpenti, ${ }_{r}^{24}$ la Lussuria è gettata in un calderone; infine una ruota è preparata per l'Ira, l'Invidia, l'Accidia.

Certo, le pene utilizzate sono poco diversificate e il principio di adattamento funziona parzialmente, soprattutto grazie a delle variazione nell'impiego del supplizio dell'ingozzamento. Ma, a dispetto di questi limiti, questo mistero sviluppa con rigore il

${ }^{20}$ Non dedica che 746 versi al Giudizio vero e proprio (Lazar 1971b: 39-41).

${ }^{21}$ Beneficiano di un trattamento di favore poiché formano una categoria indipendente dal resto laici. I falsi giudici, gli avvocati e i procuratori sono così in faccia al Giudice Supremo: si comprende che c'è una preoccupazione particolare per coloro che amministrano male la giustizia.

${ }^{22} \mathrm{Vv}$. 1825-2679: essa occupa 864 versi su un totale di 2733, circa un terzo della rappresentazione.

${ }^{23}$ È adottato l'ordine SALIGIA per la sfilata dei vizi capitali (Superbia, Avarizia, Lussuria, Invidia, Gola, Ira, Accidia). Per il dispositivo drammaturgico invece erano previste diverse fasi: innanzitutto, ciascuno per volta, ogni personaggio-Vizio era condotto verso il luogo del suo tormento; a questo veniva poi sostituito un manichino al quale l'attore prestava la sua voce. Si procedeva poi con la confessione delle malvagie azioni e alla rappresentazione della punizione. In questa fase il Vizio spesso invocava la Morte, la quale però rispondeva che, ormai, era impossibilitata ad agire. Infine il Peccato veniva gettato in un pozzo per far posto alla figura successiva. La pièce terminava con una nuova lezione, pronunciata da Satana in persona e da un messaggero angelico, a ricordo del legame tra Inferno e volontà divina: «Seigneur...le monde entier est sous ta domination, le paradis et, plus ancore, l'enfer».

${ }^{24}$ La logica iperbolica è nettamente marcata: «Ta bouche était toujours ouverte pour dévorer filets de vivande ... je te remplirai la bouche sans te arrêter et je $t^{\prime}$ assure, sur ma fois, que tes besoins seront toujours pourvus» (traduz. Lazar 1971b: 181). 
concetto di una punizione propria a ciascun peccato e dà un'importanza considerevole alla rappresentazione dei supplizi infernali.

È ugualmente interessante notare che le scene supplizianti non hanno alcuna simmetria paradisiaca: ci si accontenta di mostrare solo l'ascensione degli eletti verso il Regno dei Cieli al momento del verdetto finale del Cristo Giudice.

L'accento è messo sui peccatori e il loro supplizio produce una struttura disimmetrica che evoca, in un certo modo, l'affresco di Buffalmacco nel Camposanto di Pisa. Tuttavia, ci si deve guardare dall'interpretare questa sfasatura come una perversione dell'equilibrio giudiziario o come una rottura dell'imparzialità del Giudice. Piuttosto, essa è l'effetto (come a Pisa) di una pedagogia del pentimento. Come indicano le tirate morali pronunciate dalla personificazione dei peccati, si tratta di invitare gli spettatori a una presa di coscienza, attraverso la visione dei tormenti. Da rilevare poi l'intervento della Morte, che viene a ricordare l'urgenza della scelta. Se c'è dunque un disequilibrio è perché il dramma si rivolge agli spettatori: il rilievo dato all'Inferno più che il segno di uno scatenamento grottesco delle pulsioni popolari, è la marca di un discorso morale e didattico.

\section{Inferno e dispositivo scenico. Conclusioni}

Nella sua analisi sull'apparto drammatico medioevale, Elie Konigson (1975: 95-99) ha mostrato che l'Inferno costituisce un polo obbligato della rappresentazione sia nei drammi liturgici, svolti all'interno della chiesa, sia nei Misteri messi in scena nel contesto urbano.

In effetti, nella maggior parte delle sacre rappresentazioni, l'immagine del mondo è sempre divisa tra Inferno e Paradiso, disposti secondo gli assi cardinali; rispettivamente: «Paradiso verso levante ed Inferno verso ponente» ${ }^{25}$ (Konigson 1975: 156). Una disposizione, questa, che riprende i valori tradizionalmente attribuiti all'est e all'ovest e riproduce, nel contesto urbano, la polarizzazione dell'edificio di culto già impiegata nei drammi liturgici. Così, in veste di luogo proprio delle potenze demoniache, l'Inferno si assicura una presenza scenica anche nelle numerose rappresentazioni il cui tema non esplicita direttamente alcuna evocazione diabolica. Questa polarizzazione traduce materialmente, nello spazio teatrale, la funzione di opposizione che le pièces attribuiscono alle forze malefiche.

Per quanto concerne invece il motivo della forma della messa in scena dell'Inferno occorre, per completezza, distinguere brevemente tra i contesti francese e italiano.

Relativamente al primo ambito, si rimarca l'impiego frequente di elementi architettonici ma, in generale, è la gola che costituisce il motivo più usuale e caratteristico. Per essa, venivano usati stoffe o parti di cuoio dipinto (servivano, in particolare, ai diavoli per l'entrata o l'uscita dalla gola) giustapposti su di una struttura resistente al fuoco. Dispositivi accessori permettevano poi ai demoni di uscire dalle orecchie del mostro, di produrre fumo, odori nauseabondi e scintille (Konigson 1975: 244 e Cohen 1951: 92-99).

In Italia, la grotta costituisce l'unico elemento dello scenario della lauda umbra; e l'Inferno, come il Limbo, veniva generalmente figurato così. È solo a partire dalla metà

\footnotetext{
${ }_{25}$ Anche se l'autore usa come referenti Le Jeu de Saint Pierre et Saint Paul, rappresentato a Aix-en-Provence nel 1444 e il Mistère de Sainte Apolline (1452-1460, Konigson 1975: 125 e 97), questa disposizione, comunque, compare in tutti i drammi.
} 
del XV secolo che nei drammi viene impiegato il motivo della Gola del Leviatano (Galante Garrone 1935: 21-30; D'Ancona 1872: 478-485).

Il dato è importante perché c'è, in questa circostanza, una corrispondenza con l'ambito iconografico che, solo nel momento del secolo XV, conosce l'affermazione dell'elemento gola nel contesto infernale soprattutto dell'Italia settentrionale. Ma il parallelismo più interessante tra scena e immagine è altrove: si constata, in effetti, nelle due serie, uno sviluppo simultaneo dei supplizi infernali.

In Italia, L'Anticristo e il Giudizio Finale viene composto nel secondo quarto del XIV secolo, cioè in data prossima all'intervento di Buffalmacco a Pisa. In Francia, il Jugement dernier provenzale è rappresentato poco dopo il 1481, ossia nel momento in cui la Visione di Lazzaro conosce un'ampia diffusione nella miniatura e nell'arte monumentale. Nei due casi, la corrispondenza cronologica tra immagine e scena è sconcertante.

Tuttavia, queste considerazioni non devono indurre ad affermare che le rappresentazioni sono all'origine dell'impiego di particolari elementi (per esempio le strutture rocciose o la grotta) nelle immagini dell'Inferno. In effetti, non è che nei primi decenni del XIV secolo che le laudi danno origine a drammatizzazioni. D'altra parte il motivo delle strutture rocciose appare già negli affreschi di Giotto e Cavallini; la grotta, poi, possiede nell'arte ispirata da Bisanzio una diffusione così precoce e ampia che è là piuttosto che conviene cercare la fonte del suo uso nell'immagine infernale.

Così si può ammettere l'esistenza di un condizionamento della scena sull'iconografia sostanzialmente entro certi dati. Certo, non è escluso, per esempio, che il mantenimento o il rafforzamento del motivo della gola nell'iconografia italiana della seconda metà del XV secolo abbia qualche legame con l'attitudine visiva creata dal suo uso nelle pièces. ${ }^{26}$ Considerando il problema in un'ottica più ampia, si deve però rinunciare a una teoria che riposa soprattutto su un deprezzo radicale dell'immagine. La concordanza dello sviluppo delle rappresentazioni sceniche e iconografiche dell'Inferno, sia in Italia che in Francia, deve essere interpretato come un'evoluzione parallela dei due ambiti. Più che una coincidenza, questo pare tradurre una coerenza del campo visivo sotto forma scenica e, contemporaneamente, sotto veste iconografica.

\section{Bibliografia}

BALDelli, Ignazio (1962), «La lauda e i Disciplinati», in: Il movimento dei Disciplinati nel settimo centenario dal suo inizio, Perugia: Deputazione di Storia Patria per l'Umbria, 338-367.

Bonfantini, Mario (1942), Le sacre rappresentazioni italiane, Milano: Bompiani, 46-61. CoHEN, Gustave (1951), Histoire de la mise en scène dans le theâtre religieux français $d u$ Moyen Age, Paris: Champion.

Cohen, Gustave (1943), «The influence of mysteries on art», Gazette des-Beaux-Arts, XXIV, 327-342.

D'ANCONA, Alessandro (1872), Sacre Rappresentazioni dei secoli XIV-XVI, Firenze: Le Monnier.

D' AnConA, Alessandro (1891), Origini del teatro italiano, Torino: Einaudi.

\footnotetext{
${ }^{26}$ Allo stesso modo, alcuni motivi supplizianti sottolineano una corrispondenza tra i due ambiti. Si nota, per esempio, il frequente impiego dell'ingozzamento per gli avari. Punto di relazione particolarmente significativo è però il tormento dello spiedo per i sodomiti (in l'Anticristo e il Giudizio Universale e in numerosi affreschi), tenuto conto della rarità di questa associazione.
} 
De BARtholomaies, Vincenzo (1949), Le origini della poesia drammatica italiana, Bologna: Zanichelli.

De Bartholomaies, Vincenzo (1943), Laude drammatiche e Sacre Rappresentazioni, Firenze: Le Monnier, I, 35-52.

Galante Garrone, Virginia (1935), L'apparato scenico del dramma sacro in Italia, Torino: Rosenberg \& Sellier.

Galli, Giuseppe (1910), Laude inedite dei Disciplinati umbri, Bergamo: Istituto Italiano d'Arti Grafiche.

Konigson, Elie (1975), L'espace théatral médiéval, Paris: CNRS.

LazAr, Moshé (1971a), «L'enfer et les diables dans le théâtre médiéval italien», in: Studi di filologia romanza offerti a Silvio Pellegrini, Padova: Università degli Studi di Padova, Quaderni dell'Istituto di Filologia e Letteratura, 233-249.

LaZAr, Moshé (1971b), Le Jugement dernier. Drame provençal du XV siècle, Paris: KLINCKSIECK.

Percopo, Erasmo (1886), "Contrasto del Vivo e del Morto», Giornale Storico della letteratura italiana, VIII, n. XXI, 209-219.

RoY, Emile (1904), «Le Mystère de la Passion en France du XIV au XVI siècle», Revue Bourguignonne, III-IV, 3-189.

SHEINGORN, Pamela (1985), «For God is such a Doomsman: Origins and Development of the Theme of Last Judgment», in: Homo, memento finis: the Iconography of Last Judgment in Medieval Art and Drama, Kalamazoo, 1985, 15-58.

Le laudi drammatiche umbre delle origini. Atti del $V$ Convegno del centro di Studi sul teatro medioevale e rinascimentale (1980), Viterbo: Union printing Agnesotti.

StaAfF, Erik (1931), Le laudario de Pisa du ms.8521 de la Bibliothèque de l'Arsenal de Paris, Uppsala-Liepzig: Almqvist och Wiksell, 178-181.

Terrugia, Anna Maria (1962), «In quale momento i Disciplinati hanno dato origine al loro teatro?», in: Il movimento dei Disciplinati nel settimo centenario dal suo inizio, Perugia: Deputazione di Storia Patria per l'Umbria, 434-459.

VARANinI, Giorgio - BANFi, Luigi - Ceruti Burgio, Anna (1981), Laude cortonesi dal secolo XIII al secolo XV, Firenze: Olschki, I, 235-239.

VINTI, Anna Maria (1950), «Precisazioni sul movimento dei Flagellanti e sui maggiori laudari perugini», in: Studi di filologia italiana, VIII, 316-319.

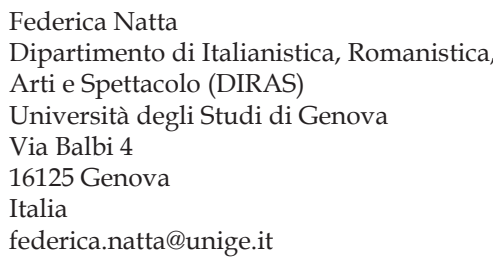

